\title{
The association of seminal plasma antioxidant levels and sperm chromatin status with genetic variants of GSTM1 and GSTP1 (Ile105Val and Ala114Val) in infertile men with oligoasthenoteratozoospermia
}

\author{
Niknam Lakpour ${ }^{\mathrm{a}}$, Azadeh Mirfeizollahi ${ }^{\mathrm{b}}$, Shirin Farivar $^{\mathrm{b}}$, Mohammad Mehdi Akhondi ${ }^{\mathrm{c}}$, \\ S. Behnam Hashemi ${ }^{\mathrm{d}}$, Naser Amirjannati ${ }^{\mathrm{c}}$, Hamed Heidari-Vala ${ }^{\mathrm{c}}$ and Mohammad Reza Sadeghi ${ }^{\mathrm{e}, *}$ \\ ${ }^{a}$ Nanobiotechnology Research Center, Avicenna Research Institute, ACECR, Tehran, Iran \\ ${ }^{\mathrm{b}}$ Department of Genetic, Faculty of Biological Sciences, Shahid Beheshti University, Tehran, Iran \\ ${ }^{\mathrm{c}}$ Reproductive Biotechnology Research Center, Avicenna Research Institute, ACECR, Tehran, Iran \\ ${ }^{\mathrm{d}}$ Iranian Tissue Bank Research and Preparation Center, Tehran University of Medical Sciences, Tehran, Iran \\ ${ }^{\mathrm{e}}$ Monoclonal Antibody Research Center, Avicenna Research Institute, ACECR, Tehran, Iran
}

\begin{abstract}
In this study we aimed to examine the effects of genetic variants of GSTM1 and GSTP1 (Ile105Val and Ala114Val) on GST activity, seminal oxidative stress and sperm chromatin status in infertile men with oligoasthenoteratozoospermia (OAT). The study population $(n=121)$ consisted of 95 infertile men with OAT and 26 controls with normozoospermia. Multiplex polymerase chain reaction (PCR) and polymerase chain reaction-restriction fragment length polymorphism (PCR-RFLP) methods were utilized to detect the aforesaid genetic variants. We measured GST activity and total antioxidant capacity (TAC) of seminal plasma by spectrophotometry. Sperm chromatin integrity and maturity were assessed using toluidine blue and chromomycin $\mathrm{A}_{3}\left(\mathrm{CMA}_{3}\right.$-positive sperm) staining, respectively. The analysis showed that subgroups of GSTM1 null and GSTP1 C/T+T/T genotypes in comparison with GSTM1 present and GSTP1 wild type (C/C) genotypes did not have statistically significant differences in both OAT or normozoospermic men considering sperm concentration and motility, percentage of $\mathrm{CMA}_{3}$-positive sperm, seminal plasma TAC, sperm chromatin integrity and GST activity. Thus, the findings of our study suggest that there are no significant associations between GSTM1 and GSTP1 polymorphisms and sperm parameters at conventional or at molecular levels including OS status, sperm chromatin integrity or maturity in Iranian infertile men with OAT and normozoospermia. However, these polymorphisms could be related to the fertility status of the studied population but not evaluated in this study.
\end{abstract}

Keywords: GSTM1, GSTP1, normozoospermia, polymorphism, oligoasthenoteratozoospermia, sperm

\section{Introduction}

Redox balance is disrupted due to imbalances in reactive oxygen species (ROS) production and their scavenging, leading ultimately to oxidative stress (OS). OS

*Corresponding author: Mohammad Reza Sadeghi, Monoclonal Antibody Research Center, Avicenna Research Institute, ACECR, Tehran, Iran. Tel.: +98 2122432020; Fax: +98 2122432021; E-mail: Sadeghi@avicenna.ac.ir. induces peroxidative damages to macromolecules in organs, tissues and cells, especially to polyunsaturated fatty acids in the cell membrane. In comparison with other cells, sperm has plenty of polyunsaturated fatty acids that make it prone to ROS-induced damages. Therefore, the protective mechanisms including antioxidant defense (enzymatic and non-enzymatic) against ROS are needed for both sperm and seminal plasma [1]. One of the enzymatic antioxidants in human sperm and seminal plasma is glutathione S-transferase (GST) that 
detoxifies products of lipid peroxidation such as aldehydes, alkanes, hydroperoxides and epoxides generated in the cell membrane. GST has at least six isoenzymes, among which implication of GSTM1 and GSTP1, in fertility-related events has been noted [2]. GSTM1 and GSTPl isoenzymes, which encode mu and pi proteins, respectively, are found on the surface of human spermatozoa [3]. These two genes have important functional polymorphisms in different racial and ethnic populations. A deletion in GSTM1 gene locus (null genotype) leads to the production of a protein with no enzymatic activity [4]. In men with unexplained infertility, the null genotype of GSTM1 increased peroxidative damage to sperm membrane and chromatin [5]. Similar results were obtained in infertile men with varicocele [6].

Two GSTP1 polymorphisms in exon 5 (Ile105 Val) and exon 6 (Ala114Val) have been reported [7]. These variants in the active site of GSTP1 are accompanied with reduction in enzyme activity [8].

Despite the importance of various GST genotypes in different diseases and their differential distribution in different populations, studies focusing on the role of GST on normal function of spermatozoa and its impairment in male sub/infertility are rather limited. Therefore, we conducted the current study to investigate the relation between seminal plasma antioxidant concentrations, sperm chromatin status, GST enzyme activity and genetic variants of GSTM1 and P1 in Iranian infertile men with severely compromised semen parameters or oligoasthenoteratozoospermia (OAT) in comparison with normozoospermic control groups.

\section{Materials and methods}

\subsection{Patients}

Semen and blood samples were obtained from men who were referred to Avicenna Infertility Clinic (AIC) in Tehran, Iran. The study sample size consisted of 121 men, which included 95 men with oligoasthenoteratozoospermia (OAT) and 26 with normozoospermia. According to WHO criteria, men were identified to have OAT if the total sperm motility was $<50 \%$, sperm count $<20 \times 10^{6} / \mathrm{ml}$ and sperm with normal shapes was $<30 \%$ [9].

Men with leukocytospermia (WBC $\geqslant 1 \times 10^{6} / \mathrm{ml}$ ), genital tract infection, congenital anomalies, highgrade varicocele, alcohol consumption, hypogonadism, history of cryptorchidism, chronic systemic disease, liver disease and positive sperm mixed-agglutination reaction test (MAR test) were excluded from the study. This study was approved by the Ethics Committee of Avicenna Research Institute, in Tehran, Iran. Written informed consent was obtained from each participant before the study.

\subsection{Sample collection and preparation}

Semen samples were obtained by masturbation after at least 48 to $72 \mathrm{~h}$ of sexual abstinence. Semen analysis was performed in accordance with WHO guidelines [9]. Each sample was aliquoted into 2 parts; part 1 was freshly used for the assessment of sperm chromatin integrity and maturity and GST activity assays, while part 2 was centrifuged at $1800 \mathrm{rpm}$ for $10 \mathrm{~min}$ utes to get clear seminal plasma for total antioxidant capacity (TAC) assay. The resultant supernatant was re-centrifuged at $4000 \mathrm{rpm}$ for 10 minutes at room temperature to remove remnants of spermatozoa and cell debris. The supernatants were stored at $-70^{\circ} \mathrm{C}$ for later TAC assay. Peripheral blood samples $(\sim 5 \mathrm{ml})$ were collected from all individuals in EDTA-containing tubes and preserved at $-20^{\circ} \mathrm{C}$ for later DNA extraction.

\subsection{Assessment of sperm chromatin integrity and maturity}

Sperm chromatin integrity and maturity were evaluated by toluidine blue (TB) and chromomycin $\mathrm{A}_{3}$ (CMA $_{3}$-positive sperm) staining tests as described by Lakpour et al. [10].

\subsection{Assessment of GST activity and TAC}

The glutathione-S-transferase activity was determined as previously described $[11,12]$. The reaction solution was constructed by mixing $980 \mu \mathrm{l}$ of $0.1 \mathrm{M}$ PBS, $10 \mu \mathrm{l}$ of $100 \mathrm{mM} \mathrm{GSH}$ and $10 \mu \mathrm{l}$ of $100 \mathrm{mM}$ 1-chloro-2,4-dinitrobenzene (CDNB). In the next step, $900 \mu \mathrm{l}$ of the aforesaid solution was added to $100 \mu \mathrm{l}$ of sperm suspension $\left(1 \times 10^{6}\right.$ sperm $)$. Reading the absorbance at $340 \mathrm{~nm}$ in a spectrophotometer thermostated at $37^{\circ} \mathrm{C}$, we calculated the difference in optical absorbance $(\Delta \mathrm{A})$ per min and reported GST activity as $\mathrm{nmol} / \mathrm{min} / 1 \times 10^{6}$ sperm.

Seminal plasma TAC was assessed by a previously described method of using a decolorization assay [13]. 


\subsection{GSTM1 and GSTP1 polymorphisms}

Genomic DNA was extracted from peripheral blood lymphocytes by salting out procedure, according to Miller et al. [14]. The presence or absence of GSTM1gene was determined using multiplex polymerase chain reaction (PCR); however analysis of GSTP1 gene polymorphism was performed by polymerase chain reaction restriction fragment length polymorphisms (PCR-RFLP). GSTM1 promoter fragments from each individual were amplified using forward primer: 5'-GAACTCCCTGAAAAGCTAA-3' and reverse primer: 5'-GTTGGGCTCAAATA TACGGTGG3'. B-globin primers (forward, 5'-CAACTTCATCCAC GTTCACC-3' and reverse, 5'-GAAGAGCCAAGGAC A GGTAC-3') were used as internal control for DNA amplification.

The multiplex PCRs were carried out in a total volume of $20 \mu \mathrm{l}$ containing $10 \mathrm{mmol} / \mathrm{l}$ Tris- $\mathrm{HCl}(\mathrm{pH} \mathrm{8.3)}$, $0.2 \mathrm{mmol} / \mathrm{l} \mathrm{MgCl} 2,50 \mathrm{mmol} / 1 \mathrm{KCl}, 0.2 \mathrm{mmol} / \mathrm{l}$ each dNTP, $0.175 \mathrm{pmol} / \mu \mathrm{l}$ ß-globin primers, $0.975 \mathrm{pmol} / \mu \mathrm{l}$ GSTM1 primers, $0.05 \mathrm{U}$ of Taq DNA polymerase (Roche) and $100 \mathrm{ng}$ of template DNA. GSTM1 PCR was performed for 35 cycles in a DNA thermal cycler using a thermal profile of denaturation at $94^{\circ} \mathrm{C}$ for $20 \mathrm{~s}$, annealing at $60^{\circ} \mathrm{C}$ for $20 \mathrm{~s}$, and primer extension at $72^{\circ} \mathrm{C}$ for $30 \mathrm{~s}$. GSTP 1 primers included forward, 5'CTATGGGAAGGACCAGCAGG-3' and reverse, 5'TGGTCTCCCACAATG-3'. GSTP1 PCRs were carried out in a total volume of $25 \mu \mathrm{l}$ containing $10 \mathrm{mmol} / \mathrm{l}$ Tris- $\mathrm{HCl}$ (pH 8.3), $1.5 \mathrm{mmol} / \mathrm{l} \mathrm{MgCl} 2,50 \mathrm{mmol} / \mathrm{l} \mathrm{KCl}$, $0.4 \mathrm{mmol} / 1$ each dNTP, $0.28 \mathrm{pmol} / \mu \mathrm{l} G S T P 1$ primers, $0.04 \mathrm{U}$ of Taq DNA polymerase (Roche) and $100 \mathrm{ng}$ of template DNA. This PCR was performed for $30 \mathrm{cy-}$ cles in a DNA thermal cycler using a thermal profile of denaturation at $94^{\circ} \mathrm{C}$ for $30 \mathrm{~s}$, annealing at $58.9^{\circ} \mathrm{C}$ for $30 \mathrm{~s}$, and primer extension at $72^{\circ} \mathrm{C}$ for $40 \mathrm{~s}$. The PCR products for GSTM1 and B-globin were separated on a $2.5 \%$ agarose gel at $80 \mathrm{~V}$ for $2 \mathrm{~h}$ and stained with $1 \mu \mathrm{g} / \mathrm{ml}$ ethidium bromide. DNA fragment sizes of the GSTM1 and B-globin genes amplified by PCR were $215 \mathrm{bp}$ and $268 \mathrm{bp}$, respectively.

Individuals with null (-) genotype of GSTM1 did not have 215 bp band while they did have the 268 bp band. The presence of PCR product for GSTP1 with a size fragment of $1069 \mathrm{bp}$ was confirmed on $1.5 \%$ agarose gel following staining by ethidium bromide $(1 \mu \mathrm{g} / \mathrm{ml})$. Subsequently, GSTP1 PCR product (1069 bp) was digested by AccI and FauI for evaluating GSTP1 polymorphisms, $313 \mathrm{~A} \rightarrow 313 \mathrm{G}$ in exon 5 and $341 \mathrm{C}>\mathrm{T}$ in exon 6 , respectively. The digestions were carried out in
Table 1

Demographic characteristics of infertile men included in this study

\begin{tabular}{lcrc}
\hline Group & Normozoospermia & OAT & P-value \\
\hline Age (year) & $37.19 \pm 6.57$ & $36.26 \pm 8.12$ & NS \\
Infertility duration (year) & $4.48 \pm 5.25$ & $5.32 \pm 7.39$ & NS \\
Smoking status & $2(9.52 \%)$ & $15(17.04 \%)$ & NS \\
\hline
\end{tabular}

OAT, oligoasthenoteratozoospermia; NS, not significant.

Table 2

The frequency of GSTM1 and P1 genotypes in OAT and normozoospermic men

\begin{tabular}{lcccc}
\hline Group & $\begin{array}{c}\text { Normozoospermia } \\
\mathrm{N}(\%)\end{array}$ & $\begin{array}{c}\text { OAT } \\
\mathrm{N}(\%)\end{array}$ & P-value & $\begin{array}{c}\text { OR } \\
(95 \% \mathrm{CI})\end{array}$ \\
\hline GSTM1 genotype & & & & \\
Null & $14(53.8)$ & $49(52.1)$ & - & - \\
Positive & $12(46.2)$ & $45(47.9)$ & 0.877 & 0.933 \\
GSTP1 genotype & & & & \\
C/T+T/T & $3(11.5)$ & $18(18.9)$ & - & - \\
C/C & $23(88.5)$ & $77(81.1)$ & 0.377 & 0.558 \\
\hline
\end{tabular}

OAT, oligoasthenoteratozoospermia; OR, odds ratio; $95 \% \mathrm{CI}, 95 \%$ confidence interval.

a total volume of $10 \mu \mathrm{l}$, once containing $1 \mu \mathrm{l}$ of NEBuffer (Biolabs, England), $0.3 \mu \mathrm{l}$ of AccI (10000 U/ml Biolabs), and $5 \mu$ l of PCR product for GSTP1 $313 \mathrm{~A}>\mathrm{G}$ and once containing $1 \mu \mathrm{l}$ of NEBuffer (Biolabs), $0.5 \mu \mathrm{l}$ of FauI (2000 U/ml Biolabs,), $3.5 \mu \mathrm{l}$ of PCR product for GSTP1341C > T.

The PCR product and its digested products were separated by electrophoresis on $10 \%$ polyacrylamide gel and were subsequently stained using silver nitrate [15]. The men with wild type (313 A/A) GSTP1 had a single 1069-bp band, those with heterozygous (313 A/G) had three $1069 \mathrm{bp}, 982 \mathrm{bp}$ and $87 \mathrm{bp}$ fragments and homozygote men $(313 \mathrm{G} / \mathrm{G})$ had two $982 \mathrm{bp}$ and $87 \mathrm{bp}$ fragments. Participants with wild type (341 C/C) GSTP1 had three 602 bp, 365 bp and 102 bp fragments, heterozygotes (341 C/T) had three $1069 \mathrm{bp}, 602 \mathrm{bp}$, and $467 \mathrm{bp}$ fragments and those with homozygote genotype (341 T/T) had two 602 bp and 467 bp fragments.

\subsection{Statistical analysis}

First, distribution of the variables was determined using one-sample Kolmogorov-Smirnov test. The comparison of frequencies for GSTM1 and GSTP1 genotypes between groups were done by chi-square test. To compare parameters with normal distribution, we used independent sample $t$ test and for parameters with nonnormal distribution, we used Mann-Whitney $U$ test. We considered $P<0.05$ as statistically significant. 
Table 3

GST polymorphisms in relation to sperm concentration, sperm motility, sperm normal morphology, percentage of $\mathrm{CMA}_{3}$-positive sperm, seminal plasma TAC, sperm chromatin damage and GST enzyme activity

\begin{tabular}{|c|c|c|c|c|c|c|c|}
\hline \multirow[t]{2}{*}{ Group } & Concentration & Motility & NM & $\mathrm{CMA}_{3}-\mathrm{ps}$ & TAC & SCD & GSTA \\
\hline & \multicolumn{7}{|c|}{ Normozoospermic men } \\
\hline \multicolumn{8}{|l|}{ GSTM1 } \\
\hline Null & $133.53 \pm 69.15$ & $54.23 \pm 23.79$ & $28.77 \pm 9.65$ & $28.16 \pm 14.52$ & $7.76 \pm 1.13$ & $35.77 \pm 20.01$ & $7.39 \pm 1.68$ \\
\hline Positive & $138.47 \pm 125.04$ & $51.25 \pm 9.32$ & $26 \pm 9.33$ & $33.45 \pm 19.09$ & $7.76 \pm 0.91$ & $31.75 \pm 19.76$ & $6.7 \pm 1.7$ \\
\hline \multicolumn{8}{|l|}{ GSTP1 } \\
\hline $\mathrm{C} / \mathrm{T}+\mathrm{T} / \mathrm{T}$ & $143.17 \pm 99.91$ & $52.5 \pm 19.25$ & $28.82 \pm 8.79$ & $31.9 \pm 17.61$ & $7.72 \pm 1.08$ & $32.85 \pm 20.11$ & $7.08 \pm 1.69$ \\
\hline $\mathrm{C} / \mathrm{C}$ & $82.66 \pm 71.49$ & 55 & $17.33 \pm 8.73^{\#}$ & $22.66 \pm 2.59$ & $7.93 \pm 0.4$ & $38.66 \pm 18.23$ & $6.95 \pm 2.18$ \\
\hline & \multicolumn{7}{|c|}{ OAT men } \\
\hline \multicolumn{8}{|l|}{ GSTM1 } \\
\hline Null & $31.93 \pm 15.97$ & $42.51(35-50)$ & $9.26 \pm 7.21$ & $40.68 \pm 20$ & $7.7 \pm 1.26$ & $35.08 \pm 17.38$ & $7.1 \pm 2.53$ \\
\hline Positive & $31.66 \pm 18.9$ & $40(16.75-50)$ & $8.75 \pm 7.2$ & $38.02 \pm 16.27$ & $7.74 \pm 1.24$ & $33.59 \pm 17.54$ & $7.47 \pm 2.9$ \\
\hline \multicolumn{8}{|l|}{ GSTP1 } \\
\hline $\mathrm{C} / \mathrm{T}+\mathrm{T} / \mathrm{T}$ & $30 \pm 14.46$ & $40(30-50)$ & $8.76 \pm 7.06$ & $39.43 \pm 19.04$ & $7.76 \pm 1.29$ & $33.87 \pm 17.87$ & $7.16 \pm 2.55$ \\
\hline $\mathrm{C} / \mathrm{C}$ & $38.28 \pm 25.33$ & $42.5(26.25-50)$ & $9.56 \pm 7.89$ & $39.87 \pm 16.1$ & $7.64 \pm 1.59$ & $36.46 \pm 15.61$ & $7.88 \pm 3.39$ \\
\hline
\end{tabular}

GST: glutathione S-transferase; TAC: total antioxidant capacity; NM: sperm normal morphology; CMA 3 -ps: percentage of CMA 3 -positive sperm; SCD: sperm chromatin damage; GSTA: glutathione S-transferase enzyme activity. \#: Compared to the GSTP1 C/T+T/T group in normozoospermic men, $p=0.045$. Data were presented in median (25th-75th percentile) and mean \pm SD.

\section{Results}

In view of age, there were no significant differences between men with OAT and normozoospermia. Other demographic characteristics and the frequencies for GSTM1 and P1 genotypes in men with OAT and normozoospermia have been presented in Tables 1 and 2, respectively.

The frequency of GSTM1 null genotype was $52.1 \%$ in men with OAT and $53.8 \%$ in men with normozoospermia $(\mathrm{OR}=0.933$; 95\% CI, 0.391-2.23; $P=$ 0.877 ) and the frequency of GSTP1 C/T+T/T genotypes were $18.9 \%$ in men with OAT and $11.5 \%$ in men with normozoopermia $(\mathrm{OR}=0.558 ; 95 \% \mathrm{CI}, 0.151-$ 2.064; $P=0.377)$. Interestingly, all individuals in the two groups were A/A in +313 site (codon 105 of GSTP 1). In the current study, there were no significant differences between men with OAT and normozoospermia with respect to the frequencies of GSTM1 and GSTP1 genotypes.

As expected, the total sperm motility $(P<0.01)$, sperm concentration and normal sperm morphology $(P<0.001)$ were significantly lower in men with OAT versus men with normozoospermia $(P<0.001)$. In addition, the percentage of $\mathrm{CMA}_{3}$-positive sperm was lower in men with normozoospermic men than men with OAT. However, the two goups of men did not have significant differences with respect to sperm chromatin integrity, GST enzyme activity, and seminal plasma total antioxidant capacity (TAC). The characteristics of GSTM1 and GSTP1 polymorphisms regarding sperm concentration, sperm normal morphology, total sperm motility, $\mathrm{CMA}_{3}$-positive sperm, seminal plasma TAC, sperm chromatin integrity and GST enzyme activity have been shown in Table 3 .

In contrast to men with OAT, the men with normozoospermia had sperm normal morphology significantly lower in the subgroup for wild type (C/C) GSTP1 genotype than in the subgroup with the $\mathrm{C} / \mathrm{T}+\mathrm{T} / \mathrm{T}$ GSTP1 genotype $(P<0.05)$. However, sperm normal morphology was not significantly different in both OAT and normozoospermic men considering the GSTMI present or null genotypes.

In addition, sperm concentration and motility, percentage of $\mathrm{CMA}_{3}$-positive sperm, seminal plasma TAC, sperm chromatin integrity and GST enzyme activities were not different in the subgroups of GSTM1 null and GSTP1 C/T $+\mathrm{T} / \mathrm{T}$ genotypes versus the subgroups of GSTM1 present and GSTP1 wild type (C/C) genotypes in both groups of men with OAT and normozoospermia.

\section{Discussion}

Seminal oxidative stress created via over-production of ROS by means of white blood cells (WBCs), dead and immature spermatozoa is one of the important causes of male sub/infertility. Antioxidant defense systems, especially enzymatic ones, play a critical role in preventing or creation of OS. GSTs, as a member of enzymatic defense, attenuate OS through binding reduced GSH to toxic oxidants. Moreover, interactions between GST genes and deleterious environmental exposures have been addressed by exhibiting the presence of GST 
on the head of spermatozoa and its role in detoxifying certain carcinogens [16].

GST gene polymorphisms and male sub/infertility is very dependent on ethnicity. Hence, the present study focused on genotypes of two isoenzymes of GSTM1 and GSTP1. In GSTP1, substitution of Val instead of Ile and Ala in exon 5 (Ile105Val) and exon 6 (Ala114Val) significantly reduced enzyme activity. Therefore, simultaneous evaluation of these two polymorphisms in male infertility is a novel idea that has not been addressed yet.

According to our findings, frequency of GSTM1 and GSTP1 genotypes (both sites) were not significantly different between normozoospermic men and men with OAT. This finding had been previously confirmed in fertile men with good semen quality [17]. Olshan et al. showed that gene polymorphisms of GSTM1, GSTT1, and GSTZ1 are not correlated with sperm quality. As a result, there seems to be no statistically significant relation between GSTs genotypes and changes in classical semen parameters in fertile and infertile men. Previous studies performed in different countries, including China (men with varicocele vs. fertile men; GSTM1 and P1) [6], Turkey (men with varicocele vs. fertile men; GSTM1) [18], and Russia (idiopathic infertile men vs. fertile men; GSTM1) [19] have shown no significant differences between fertile men and men with varicocele or infertility. However, Tirumala, et al. indicated that the frequency of GSTM1 null genotype is significantly lower in fertile than infertile men [20]. Additionally, Finotti et al. also showed that semen quality of men with idiopathic infertility decreases in GSTM1/GSTT1 null genotypes [21]. Likewise, two more studies in the latter relation have been performed in Iran. The first, performed by Safarinejad et al., had surprising and unexpected results [22], but the second one, performed by Salehi et al., showed a correlation between idiopathic male infertility and GSTT1 and GSTM1 null deletions in men from Northern Iran [23]. Contrary to the study by Safarinejad et al. we found no individual with hetero- or homozygote genotypes in codon 105 of GSTP1 (GSTP1 $313 \mathrm{~A} / \mathrm{G}$ and $313 \mathrm{G} / \mathrm{G}$ polymorphisms). For this reason, we checked the accuracy of restriction enzyme (AccI) activity by performing RFLP on the other nucleotide sequence that had a cutting site for AccI.

Contrary to our expectation, we found a positive correlation between sperm normal morphology and GSTP1 C/T+T/T genotypes only in normozoospermic men. Although, sperm with normal morphology was higher in OAT men with GSTP1 C/T+T/T genotypes than in OAT men with GSTP1 C/C genotype, but this difference was not statistically significant. In connection to this finding, two inferences can be suggested, the first GSTP1 C/T+T/T genotypes are associated with sperm normal morphology (a protective role in normal spermatogenesis) and the second, increasing sample size in normozoospermic men could change this result. Therefore, any ultimate conclusions in this regard would be premature and further studies would be necessary.

Similar to Aydemir et al., we did not find any difference between GSTM1 and GSTP1 genotypes in total antioxidant capacity and GST enzyme activity [5]. Therefore, these polymorphisms seem not to affect GST activity and, consequently, antioxidant concentration in the seminal plasma of men with OAT or normozoospermic men.

Reduction of enzyme activity in one or two genotypes is compensated by other GST isoenzymes, including GSTT1, GSTA1, and GSTM2. Aydemir et al. found that GSTM1 null genotype might negatively affect spermatozoa by increasing the oxidative stress in men with idiopathic infertility [5]. However, our findings did not confirm this concept in men with OAT or normozoospermia.

Since the studied polymorphisms did not affect classical sperm parameters, we decided to evaluate their effect on human spermatozoa at molecular level, including sperm chromatin integrity and maturity. In this regard, the present study showed sperm chromatin damage and maturity were not different in both men with OAT or normozoospermia; regarding GSTM1 null and GSTP1 C/T+T/T genotypes compared to GSTMpresent and GSTP1 C/C genotypes. Aydemir et al. mentioned that sperm is further susceptible to oxidative damage in idiopathic infertility with GSTM1 null genotype [5]. In addition, Tang et al. showed that in men with varicocele, the GSTMI and GSTT1 null genotypes lead to sperm oxidative damage but GSTP1 alleles (codon 105) do not affect OS status of semen and, subsequently, oxidative damage of spermatozoa [6].

Finally, it should be mentioned that our study had some limitations. First, we did not cover fertile men in our study. Moreover, the normozoospermic group had a small sample size. Additionally, TAC-ROS score could show OS status better than TAC.

\section{Conclusion}

In conclusion, the findings of this study suggests that there are no significant associations between GSTM1 
and GSTP1 (codon of 114) polymorphisms and sperm parameters at classical or at molecular levels, including OS status, sperm chromatin integrity or sperm chromatin maturity in male Iranians with OAT and normozoospermia. However, these polymorphisms may be related to the fertility status of the studied men not evaluated in this study.

\section{Acknowledgements}

The authors would like to thank Ms. Elham Savadi Shirazi for her contribution in collecting samples in Avicenna Infertility Clinic.

\section{References}

[1] Agarwal A, Saleh RA, Bedaiwy MA. Role of reactive oxygen species in the pathophysiology of human reproduction, Fertil Steril 79 (2003), 829-43.

[2] Gopalakrishnan B, Aravinda S, Pawshe CH, Totey SM, Nagpal S, Salunke DM, et al. Studies on glutathione S-transferases important for sperm function: evidence of catalytic activityindependent functions. Biochem J 15 (1998), 231-41.

[3] Jiang S, Yu L, Cheng J, Leng S, Dai Y, Zhang Y, et al. Genomic damages in peripheral blood lymphocytes and association with polymorphisms of three glutathione S-transferases in workers exposed to formaldehyde, Mutat Res 695 (2010), 9-15.

[4] Cotton SC, Sharp L, Little J, Brockton N. Glutathione Stransferase polymorphisms and colorectal cancer: a HuGE review. Am J Epidemiol 151 (2000), 7-32.

[5] Aydemir B, Onaran I, Kiziler AR, Alici B, Akyolcu MC. Increased oxidative damage of sperm and seminal plasma in men with idiopathic infertility is higher in patients with glutathione S-transferase Mu-1 null genotype, Asian J Androl 9 (2007), 108-15.

[6] Tang K, Xue W, Xing Y, Xu S, Wu Q, Liu R, et al. Genetic polymorphisms of glutathione S-transferase M1, T1, and P1, and the assessment of oxidative damage in infertile men with varicoceles from northwestern China, Journal of andrology 33 (2012), 257-63.

[7] Board PG, Webb GC, Coggan M. Isolation of a cDNA clone and localization of the human glutathione S-transferase 3 genes to chromosome bands 11q13 and 12q13-14. Ann Hum Genet 53 (1989), 205-13.

[8] Ali-Osman F, Akande O, Antoun G, Mao JX, Buolamwini J. Molecular cloning, characterization, and expression in Escherichia coli of full-length cDNAs of three human glutathione S-transferase Pi gene variants. Evidence for differential catalytic activity of the encoded proteins, J Biol Chem 11 (1997), 10004-12.
[9] WHO. World Health Organization Laboratory Manual for the Examination of Human Semen and Sperm-Cervical Mucus Interaction. (Cambridge University Press, Cambridge, UK, 1999).

[10] Niknam Lakpour, Reda Z Mahfouz, Mohammad Mehdi Akhondi, Ashok Agarwal, Hadi Kharrazi, Hojjat Zeraati NA, Mohammad Reza Sadeghi. Relationship of seminal plasma antioxidants and serum male hormones with sperm chromatin status in male factor infertility, Systems Biology in Reproductive Medicine 58 (2012), 236-44.

[11] Mannervik B. The isoenzymes of glutathione transferase. Adv Enzymol Relat Areas Mol Biol 57 (1985), 357-417.

[12] Coles B, Ketterer B. The role of glutathione and glutathione transferases in chemical carcinogenesis, Crit Rev Biochem Mol Biol 25 (1990), 47-70.

[13] Re R, Pellegrini N, Proteggente A, Pannala A, Yang M, RiceEvans C. Antioxidant activity applying an improved ABTS radical cation decolorization assay, Free Radic Biol Med 26 (1999), 1231-7.

[14] Miller SA, Dykes DD, Polesky HF. A simple salting out procedure for extracting DNA from human nucleated cells. Nucleic Acids Res 16 (1988), 1215.

[15] Benbouza H JJ, Baudin JP, Mergeai G. Optimization of a reliable, fast, cheap and sensitive silver staining method to detect SSR markers in polyacrylamide gels, Biotechnology, Agronomy, Society and Environment 10 (2006), 77-81.

[16] Rubes J, Selevan SG, Sram RJ, Evenson DP, Perreault SD. GSTM1 genotype influences the susceptibility of men to sperm DNA damage associated with exposure to air pollution, Mutat Res 625 (2007), 20-8.

[17] Olshan AF, Luben TJ, Hanley NM, Perreault SD, Chan RL, Herring AH, et al. Preliminary examination of polymorphisms of GSTM1, GSTT1, and GSTZ1 in relation to semen quality, Mutation res 688 (2010), 41-6.

[18] Acar H, Kilinc M, Guven S, Inan Z. Glutathione S-transferase M1 and T1 polymorphisms in Turkish patients with varicocele, Andrologia 44 (2011), 34-7.

[19] Polonikov AV, Yarosh SL, Kokhtenko EV, Starodubova NI, Pakhomov SP, Orlova VS. The functional genotype of glutathione S-transferase T1 gene is strongly associated with increased risk of idiopathic infertility in Russian men, Fertil Steril 94 (2010), 1144-7.

[20] Tirumala Vani G, Mukesh N, Siva Prasad B, Rama Devi P, Hema Prasad M, Usha Rani P, et al. Role of glutathione Stransferase Mu-1 (GSTM1) polymorphism in oligospermic infertile males, Andrologia 42 (2010), 213-7.

[21] Finotti AC, Costa ESRC, Bordin BM, Silva CT, Moura KK. Glutathione S-transferase M1 and T1 polymorphism in men with idiopathic infertility, Genet Mol Res 8 (2009), 1093-8.

[22] Safarinejad MR, Shafiei N, Safarinejad S. The association of glutathione-S-transferase gene polymorphisms (GSTM1, GSTT1, GSTP1) with idiopathic male infertility, J Hum Genet 55 (2010), 565-70.

[23] Salehi Z, Gholizadeh L, Vaziri H, Madani AH. Analysis of GSTM1, GSTT1, and CYP1A1 in idiopathic male infertility, Reprod Sci 19 (2012), 81-5. 


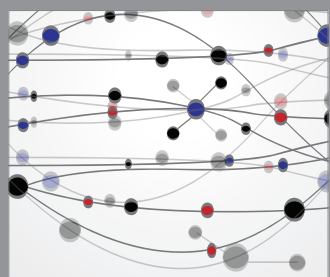

The Scientific World Journal
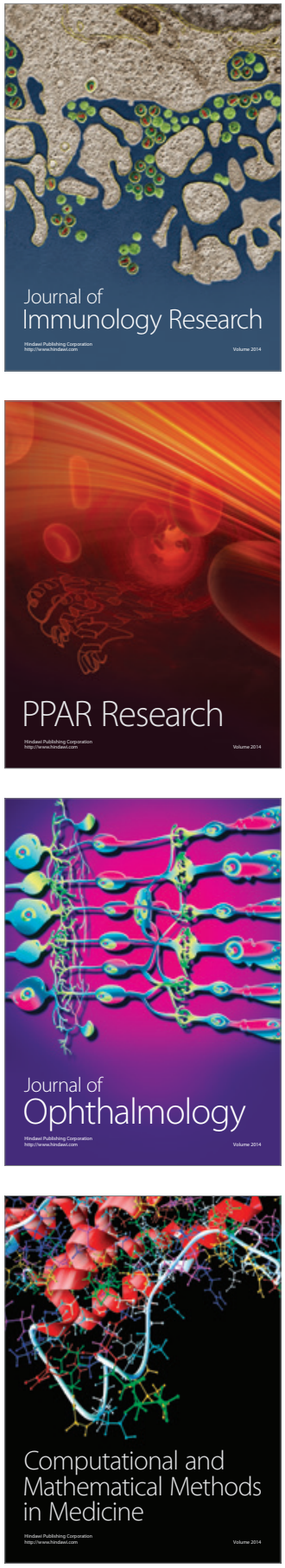

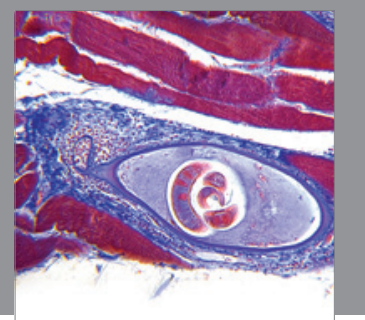

Gastroenterology

Research and Practice
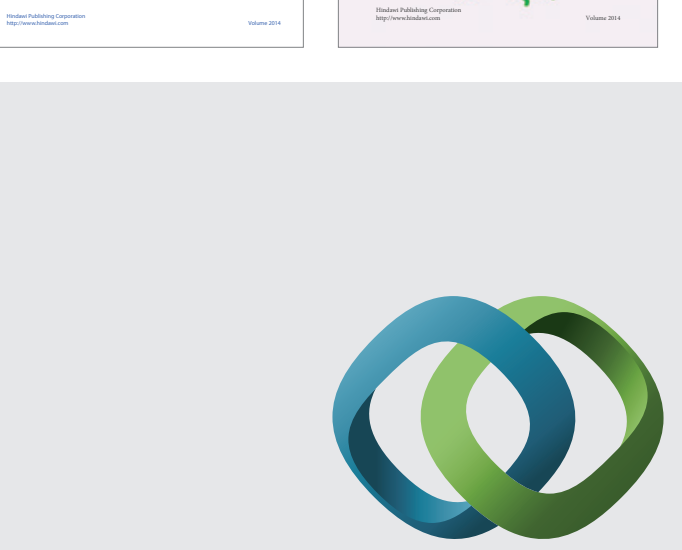

\section{Hindawi}

Submit your manuscripts at

http://www.hindawi.com
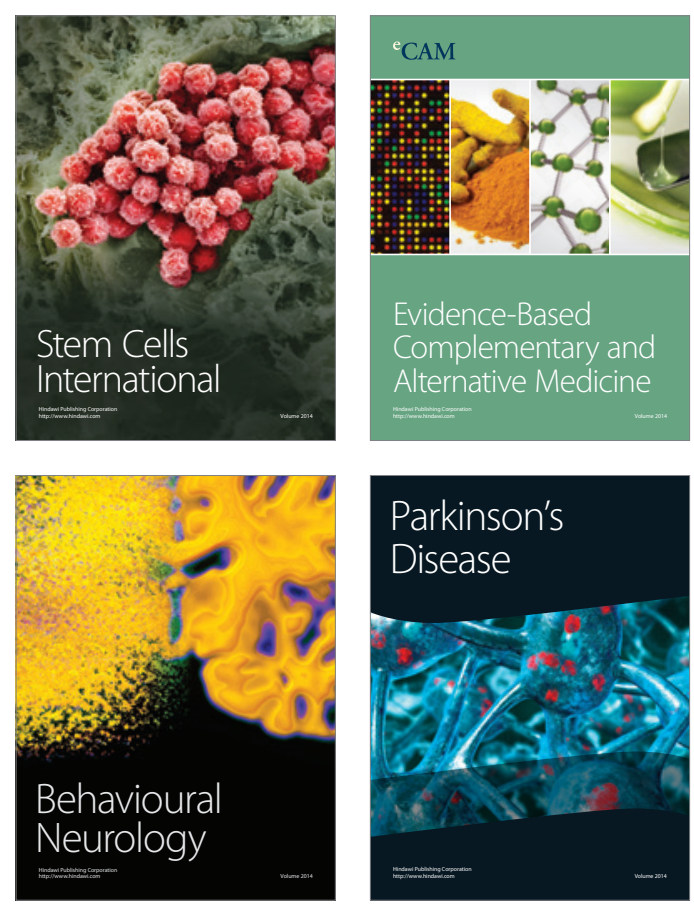

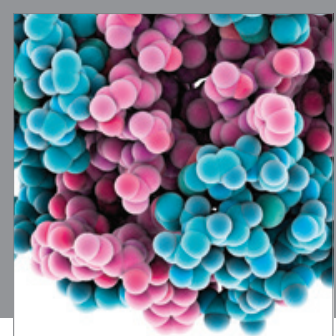

Journal of
Diabetes Research

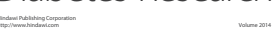

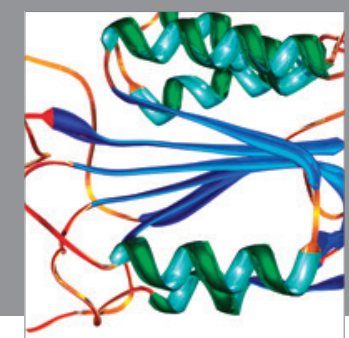

Disease Markers
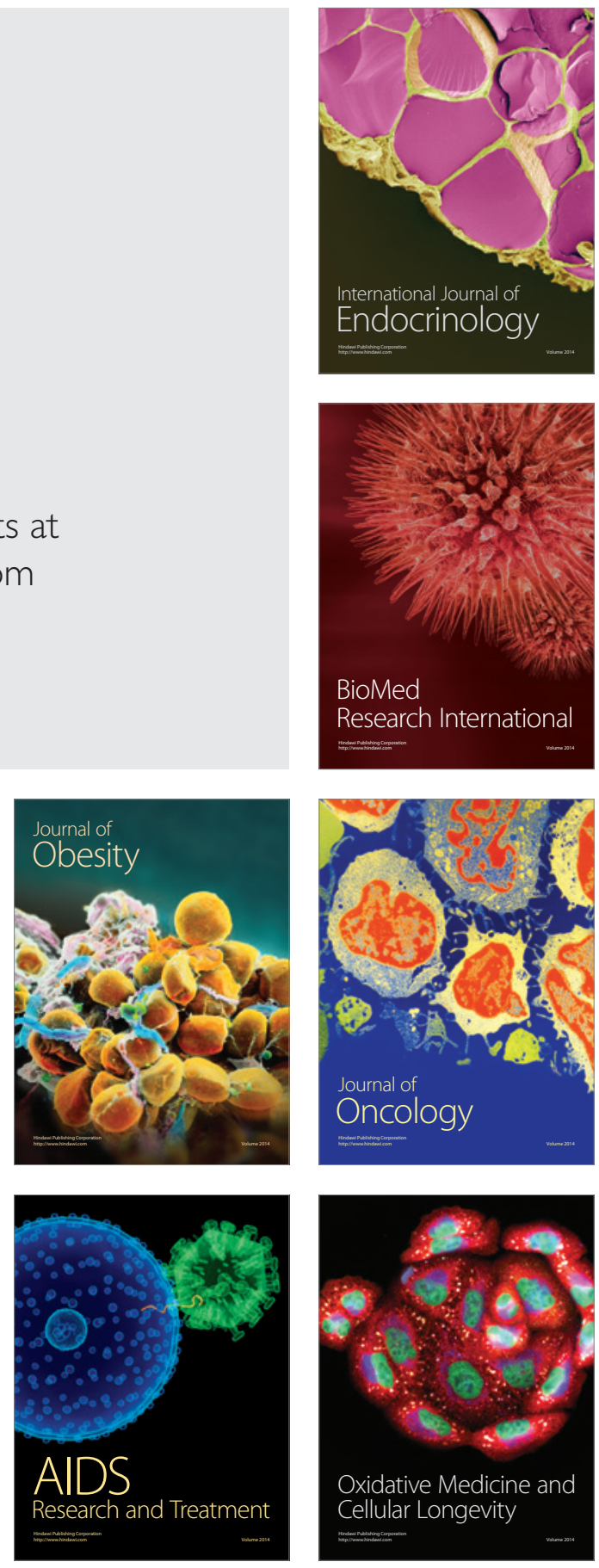